REVISTA EUREKA
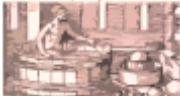

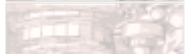

Revista Eureka sobre Enseñanza y Divulgación de las Ciencias

ISSN: 1697-011X

revista.eureka@uca.es

Universidad de Cádiz

España

\title{
La cultura del agua en los libros de texto
}

Benarroch Benarroch, Alicia; Castro Velásquez, Freddy Enrique; Clavijo Cuervo, Vilma Jannett; RamírezSegado, Alejandra

La cultura del agua en los libros de texto

Revista Eureka sobre Enseñanza y Divulgación de las Ciencias, vol. 19, núm. 1, 2022

Universidad de Cádiz, España

Disponible en: https://www.redalyc.org/articulo.oa?id=92068491019

DOI: https://doi.org/10.25267/Rev_Eureka_ensen_divulg_cienc.2022.v19.i1.1501 
Educación científica y sostenibilidad

\section{La cultura del agua en los libros de texto}

Water Culture in the textbooks

Alicia Benarroch Benarroch

Departamento de Didáctica de las Ciencias Experimentales, Facultad de Ciencias de la Educación y del Deporte de

Melilla, Universidad de Granada, España

aliciabb@ugr.es

iD https://orcid.org/0000-0003-0316-0141

Freddy Enrique Castro Velásquez

Secretaria de Educación Distrital de Bogotá, Colombia

freddycastro@correo.ugr.es

iD https://orcid.org/0000-0002-8611-5460

Vilma Jannett Clavijo Cuervo

Secretaria de Educación Distrital de Bogotá, Colombia

vjclavijo@educacionbogota.edu.co

iD https://orcid.org/0000-0001-9637-7360

Alejandra Ramírez-Segado

Departamento de Didáctica de las Ciencias Experimentales, Facultad de Ciencias de la Educación y del Deporte de

Melilla, Universidad de Granada, España

alermzsgd@ugr.es

iD https://orcid.org/0000-0002-9547-6647

DOI: https://doi.org/10.25267/

Rev_Eureka_ensen_divulg_cienc.2022.v19.i1.1501

Redalyc: https://www.redalyc.org/articulo.oa? $\mathrm{id}=92068491019$

Recepción: 02 Septiembre 2021

Revisado: 22 Octubre 2021

Aprobación: 04 Noviembre 2021

\section{ReSUMEN:}

La cultura del agua o cultura hídrica viene determinada por las costumbres, las actitudes y los valores, tanto individuales como compartidos socialmente, que adoptamos las personas respecto al agua. En este trabajo, se afronta el análisis de la cultura del agua que se transmite en 20 libros de texto españoles y colombianos diseñados para estudiantes de 14-15 años de edad. Con este fin, se elaboró un instrumento taxonómico fundamentado en las corrientes de la Nueva Cultura del Agua frente a la tradicional. Este instrumento fue útil en la caracterización de las Unidades de Enseñanza-Aprendizaje identificadas en los libros de texto. Los resultados indican una alta dispersión en la imagen cultural que sobre el agua ofrecen los libros, tanto españoles como colombianos, aunque globalmente se manifiesta una tímida tendencia hacia la cultura más tradicional.

Palabras Clave: Libros de texto, Cultura del agua, Cultura hídrica, Nueva Cultura del Agua, Sostenibilidad.

\section{ABSTRACT:}

Water culture is determined by customs, attitudes and values, both individual and socially shared, that people adopt with respect to water. In this paper, we analyse the water culture transmitted in 20 Spanish and Colombian textbooks designed for students aged 14-15 years old. To this end, a taxonomic instrument was developed based on the differences between the New Water Culture and the traditional one. This instrument was useful in characterising the Teaching-Learning Units identified in the textbooks. The results indicate a high dispersion in the cultural image of water in both Spanish and Colombian textbooks, although overall there is a timid tendency towards the more traditional culture.

KEYWORDs: Textbooks, Water, Water culture, New Water Culture, Sustainability. 


\section{INTRODUCCIÓN}

En la actualidad, estamos viviendo lo que los científicos llaman el Antropoceno, una nueva era geológica en la que el ser humano se ha convertido en la fuerza dominante que moldea el entorno natural. Bajo el mal llamado "progreso", hemos asumido la creencia de que la Tierra es nuestra para explotarla y permitir un crecimiento infinito. Pero la Tierra ha empezado a expresar sus límites y a mostrar los primeros síntomas de una multicrisis ambiental e hídrica.

La crisis hídrica es una situación que se produce cuando la disponibilidad de agua no contaminada dentro de una región es inferior a su demanda, entendida esta como la suma total del agua consumida tanto en el ámbito doméstico como agroindustrial. El ranking del Instituto de Recursos Mundiales clasificó en 2019 por su nivel de estrés hídrico los 164 países del mundo en cinco grupos. España queda situada en el puesto 28, entre los países con alto nivel, y con zonas, como la cuenca del Segura, donde es extremadamente alto. Colombia, otro país directamente involucrado en esta investigación, se sitúa, en cambio, en la posición 118 , y, por tanto, entre los países con menor nivel de estrés hídrico (WRI 2019).

¿Cómo afrontamos esta situación desde la práctica? Como afirma Sutoris (2021), ante estos avisos y amenazas, hemos de reconstruir la relación con nuestro planeta. Hemos de cambiar la mentalidad colectiva y educar a nuestros hijos en la humildad y en el respeto a la naturaleza. En relación con el agua, se trataría de reflexionar sobre las costumbres, las actitudes y los valores, tanto individuales como compartidos socialmente y concederle el lugar patrimonial y hegemónico que se merece. En definitiva, se trata de cambiar la cultura del agua.

Los procesos educativos formales pueden ser los cimientos de estos cambios culturales comunitarios. En ellos, se puede afrontar la problemática ambiental, plantear alternativas, y valorar, además de las aportaciones e innovaciones tecnológicas, las que se asocian a los cambios de nuestras mentalidades y concepciones con la creencia de que los recursos naturales son ilimitados y sobre el consumismo de bienes y de posesiones materiales.

Los libros de texto son los recursos didácticos más utilizados en las aulas y no deben ni pueden ser concebidos únicamente como meros instrumentos didácticos ya que también contienen una dimensión ideológica, pues "reflejan una concepción del mundo, unos valores y unas prioridades" (Occelli y Valeiras 2013, p.133) que conforman un cuerpo de conocimientos legitimizado por ser, para los profesores, la interpretación del currículum oficial más cercana de su práctica (López-Valentín y Guerra-Ramos 2013). En consecuencia, participan de la enorme responsabilidad de ser los agentes mediadores de los cambios culturales que las sociedades pretendan realizar.

En este trabajo, se afronta el análisis de la cultura hídrica o cultura del agua que se transmite en los libros de texto españoles y colombianos diseñados para estudiantes de 15 años de edad. Asimismo, se busca indagar en las eventuales diferencias entre ambos países, teniendo en cuenta la diversidad en sus niveles de estrés hídrico, y evaluar si el peso de la cultura hídrica del agua es diferente en las asignaturas donde curricularmente se considera.

A través de un instrumento taxonómico fundamentado en las corrientes de la Nueva Cultura del Agua (NCA, en adelante) frente a la tradicional, se pretende caracterizar los libros de texto y desvelar la imagen que estamos transmitiendo a nuestros estudiantes en relación con esta sustancia fundamental para la vida.

\section{MARCO TEÓRICO}

¿Es posible analizar la cultura del agua en los libros de texto? ¿Qué entendemos por cultura del agua? ¿Es posible identificar sus características de una manera que permita discriminar en los libros de texto entre una cultura más tradicional y una cultura más transformadora en cuanto a las relaciones del ser humano con los recursos hídricos...? Estas son algunas de las cuestiones que hemos ido abordando en este trabajo, y a las que 
nos iremos refiriendo a continuación, a medida que expongamos las decisiones más relevantes que fueron tomadas para resolverlas.

La cultura es la forma de ser de un pueblo y puede describirse como las características y los conocimientos de un grupo de personas que engloba valores, creencias, costumbres y tradiciones (Nnebuihe 2019) y constituye un poderoso factor de innovación económica y social, así como de movilización de proyectos de desarrollo sostenible (UNESCO 2010).

En este sentido, la cultura hídrica o cultura del agua es relevante en la identificación e implementación de alternativas para afrontar la problemática social y ambiental relacionada con los recursos hídricos. Aunque en el año 2015, se aprobó la Agenda 2030 de desarrollo sostenible, con un objetivo destinado al tema de los recursos hídricos, actualmente la humanidad se enfrenta a una crisis hídrica sin precedentes y se estima que, en el año 2030, casi la mitad de la población mundial, vivirá en zonas con un elevado estrés hídrico (WWDR 2020).

Las estrategias que distintos países y organismos sociales y políticos han puesto en marcha para resolver el problema de agua han sido muy diversas. Robles y Maldonado (2018) citan la utilización de los medios de comunicación para sensibilizar a la ciudadanía; la aplicación de tarifas reales; la implementación de tecnologías más eficientes para el mejor aprovechamiento del agua; las mejoras infraestructurales; y la implementación de proyectos de gamificación para mejorar los hábitos y actitudes de los ciudadanos frente al agua. Aunque los porcentajes de éxito han sido variados en referencia al aprovechamiento del agua, como el $40 \%$ en el Reino Unido y el 12\% en España, destacan sobre todo el éxito obtenido por Israel, considerado como el país número 1 en gestión sostenible del recurso hídrico, con un $75 \%$ de aprovechamiento del agua, tras la implementación de una estrategia multifacética cuyo punto clave reside en la educación (Shcwab 2017).

La educación, por tanto, debe ser considerada como una corriente de integración, desarrollo y sostenibilidad, que desempeña un papel central en la formación de la identidad personal y colectiva en la producción de una ciudadanía basada en los principios de respeto a la vida, la dignidad humana y la diversidad cultural (Nnebuihe 2019).

El agua es uno de los contenidos más extendidos en el currículo escolar de todas las etapas educativas (Ramírez-Segado et al. 2021) y presuponemos que los libros de texto se hacen eco de esta profusión. Los libros de texto siguen siendo uno de los recursos educativos más utilizados en el aula (Cañal et al. 2016, Fernández y Caballero 2017, Martínez y Alarcón 2016), y son pocos los centros educativos que los sustituyen por mecanismos de información más flexibles y abiertos (Perales 2019), pues es manifiesta la preferencia del profesorado por este tipo de material didáctico en menoscabo de la elaboración de material propio (Martínez y Rodríguez 2010), así como las subvenciones que con frecuencia reciben las familias para su adquisición (Perales 2019).

Aunque hay autores, como Fernández y Caballero (2017), que resaltan el valor del libro de texto como el reflejo del estado del conocimiento que se tiene en las diferentes disciplinas, esto no siempre es así, y se transmiten conceptos e ideas descartadas por la comunidad científica (Parcerisa 1996). En el caso del agua, existen numerosos artículos que ponen de manifiesto la existencia de errores conceptuales y/o visiones parciales del ciclo del agua (Fernández-Ferrer 2009, Santana et al. 2016), además de la desconexión del ciclo con la acción del ser humano (Antoraz y Martínez 2003). Asimismo, las relaciones de las ciudades con sus ríos tienen escasa presencia en los libros de texto y vienen marcadas por el valor económico del agua, el río como amenaza y el ahorro doméstico como único compromiso (Cuello y García 2019). La presencia de errores conceptuales o de sesgos valorativos en los textos influye en la manera en la que los estudiantes interpretan la realidad, además de carecer de la objetividad necesaria para proporcionar una enseñanza crítica y abierta (Cañal et al. 2016). En consecuencia, los conocimientos y valores sesgados contemplados en los textos arraigan y crecen en el alumnado y se consolidan en el profesorado de manera que se genera un sistema de creencias muy difíciles de cambiar (Cañal et al.2016). Si ello ocurriera con la cultura del agua, no se estaría 
contribuyendo a generar un cambio en la cultura hídrica, algo que es tan necesario en la situación de crisis hídrica global.

\section{El nacimiento de la Nueva Cultura del Agua}

Una de las cuestiones teóricas más importantes que se plantea en este trabajo es la identificación de las características que deberían distinguir una cultura del agua alternativa a la tradicional y que, llevada al ámbito educativo, fuera generadora de unos conocimientos, comportamientos y actitudes transformadores de las relaciones de las personas con el medio hídrico.

En el año 1998, surge en España la Fundación de la Nueva Cultura del Agua (FNCA, en adelante), un movimiento social que trata de dar respuesta a la obsolescencia de los modelos de gestión hídrica tradicional, basados en la oferta ilimitada de agua. Impulsada por la FNCA, en el año 2005, y tras el visto bueno de más de 100 científicos de 16 países diferentes, se publica la Declaración Europea por la Nueva Cultura del Agua, con fin de exigir al parlamento europeo el endurecimiento de las medidas para asegurar que los países de la Unión Europea cumpliesen la Directiva Marco de Agua (Comunidades Europeas 2000), lo que supuso la entrada en escena del movimiento defensor de la NCA después de casi diez años de existencia (Pérez 2015). La NCA defiende un profundo cambio hacia unas nuevas sensibilidades sociales y ambientales que puedan garantizar la sostenibilidad de los ecosistemas acuáticos y el acceso efectivo de los derechos humanos al agua potable y saneamiento básico, impulsar una nueva forma de gobernanza participativa e instaurar en una ciudadanía global derechos universales (Arrojo 2009).

Las principales ideas que permiten diferenciar entre la visión tradicional del agua, denominada vieja cultura del agua, y la visión desde un desarrollo sostenible que promueve la NCA, se pueden expresar a través de siete contextos sintetizados en Benarroch et al. (2021) y reproducidos, con ligeras modificaciones, en la Tabla 1. Estos contextos fueron el punto de partida para la generación del instrumento que se utilizó para el análisis de los libros de texto en este trabajo. 
TABLA 1

Contextos que diferencian la NCA de la tradicional

\begin{tabular}{|c|c|c|}
\hline Contextos & Vieja Cultura del Agua & Nueva Cultura del Agua \\
\hline & Desequilibrio hídrico & Equilibrio natural \\
\hline 1 & $\begin{array}{l}\text { 1. El agua es escasa. El agua dulce que hay } \\
\text { en la Tierra es insuficiente. La escasez } \\
\text { del agua se debe al desequilibrio hídrico. } \\
\text { 2. El agua es un recurso ilimitado y } \\
\text { renovable. }\end{array}$ & $\begin{array}{l}\text { 1. El agua no es escasa, es suficiente si la } \\
\text { cuidamos. La eventual escasez de agua se } \\
\text { debe a una mala gestión y al abuso en su uso } \\
\text { y su consumo. } \\
\text { 2. El agua es un recurso renovable cuando se } \\
\text { controla cuidadosamente su uso, } \\
\text { tratamiento, liberación, circulación. De lo } \\
\text { contrario es un recurso no renovable. }\end{array}$ \\
\hline 2 & $\begin{array}{l}\text { 2. El agua es una mercancía, un bien } \\
\text { económico. }\end{array}$ & $\begin{array}{l}\text { Agua como activo ecosocial } \\
\text { 1. El agua tiene distintas funciones: una parte } \\
\text { muy pequeña es un recurso básico, pero } \\
\text { toda ella tiene un valor patrimonial. } \\
\text { 2. El agua no es sólo un bien económico, sino } \\
\text { que también es fundamental para la } \\
\text { supervivencia y el futuro de nuestro planeta. }\end{array}$ \\
\hline & Gobernabilidad del agua & Gobernanza del agua \\
\hline 3 & $\begin{array}{l}\text { 1. La responsabilidad del agua recae en las } \\
\text { autoridades institucionales y gobiernos. } \\
\text { 2. La gestión tradicional del agua necesita } \\
\text { cambios urgentes. }\end{array}$ & $\begin{array}{l}\text { 1. La responsabilidad del agua recae en toda la } \\
\text { ciudadanía. Se requieren programas } \\
\text { formativos que comiencen en las escuelas. } \\
\text { 2. La gestión sostenible del agua es posible y ya } \\
\text { hay propuestas concretas. }\end{array}$ \\
\hline 4 & $\begin{array}{l}\text { Gestión de la oferta } \\
\text { 1. Se requiere aumentar la cantidad de agua } \\
\text { dulce disponible. } \\
\text { 2. Las obras hidráulicas (embalses, } \\
\text { trasvases, desalinizadoras, etc.) son } \\
\text { necesarias para conseguir más agua. }\end{array}$ & $\begin{array}{l}\text { Gestión de la demanda } \\
\text { 1. Se debe reducir el consumo y no almacenar } \\
\text { más agua. } \\
\text { 2. Las obras hidráulicas son dañinas para el } \\
\text { medio ambiente. Es mejor poner todo el } \\
\text { empeño en disminuir la demanda de agua. }\end{array}$ \\
\hline 5 & $\begin{array}{l}\text { Coste-beneficio } \\
\text { 1. El régimen tarifario del agua no } \\
\text { diferencia entre agua-vida y las restantes } \\
\text { funciones del agua. } \\
\text { 2. Quien contamina, no paga. }\end{array}$ & $\begin{array}{l}\text { Coste-efectividad } \\
\text { 1. El régimen tarifario del agua debe diferenciar } \\
\text { entre agua-vida (gratis) y las restantes } \\
\text { funciones del agua (tarifas, canon...). } \\
\text { 2. Quien contamina, debe descontaminar o } \\
\text { dejar de contaminar }\end{array}$ \\
\hline 6 & $\begin{array}{l}\text { Agua como derecho humano } \\
\text { 1. El agua es un derecho humano que debe } \\
\text { ser satisfecho por los gobernantes. } \\
\text { 2. Hay poblaciones que carecen del agua- } \\
\text { vida; los gobiernos deben } \\
\text { responsabilizarse de ello. }\end{array}$ & $\begin{array}{l}\text { Agua como deber humano } \\
\text { 1. Sólo una pequeña parte del agua es agua- } \\
\text { vida. El resto tiene aparejados unos deberes } \\
\text { (obligaciones de ahorro y de costes). } \\
\text { 2. Hay poblaciones que carecen del agua-vida. } \\
\text { Todos debemos responsabilizarnos. }\end{array}$ \\
\hline 7 & $\begin{array}{l}\text { 2. El consumismo de bienes y los hábitos } \\
\text { alimentarios no afectan al consumo de } \\
\text { agua. }\end{array}$ & $\begin{array}{l}\text { Consumerismo } \\
\text { 1. El ahorro de agua es una prioridad a todos } \\
\text { los niveles, doméstico, agrícola, industrial, } \\
\text { etc. } \\
\text { 2. El consumismo de bienes y los hábitos } \\
\text { alimentarios están intimamente relacionados } \\
\text { con el consumo de agua. Debemos ser } \\
\text { consumidores responsables. }\end{array}$ \\
\hline
\end{tabular}

(adaptado de Benarroch et al. 2021) 


\section{Metodología}

\section{Instrumento}

El instrumento utilizado para el análisis de los libros de texto se muestra en el Anexo 1. Se trata de una estructura taxonómica que mantiene los siete contextos de la Tabla 1. Asimismo, la mayoría de los subcontextos se corresponden con los apartados descritos en cada uno de los contextos. Solo hay una excepción, relacionada con el contexto 2, en el que se han distinguido tres subcontextos, y no solo dos como aparecen en la Tabla 1, al considerar que podría ser muy representativo de la imagen cultural del agua que transmiten los libros de texto, el análisis de las actividades de reflexión crítica sobre la importancia del agua para la vida. Esto hizo que, en lugar de 14, fuera 15 el total de los subcontextos.

Para cada subcontexto, se trató de generar una escala cuantitativa que permite asignar un valor numérico entre el uno y el tres a cada una de las unidades de enseñanza-aprendizaje (UEA) identificadas en los libros de texto, con el fin de caracterizar su potencial formativo y su congruencia con la NCA. De este modo, el valor de uno indica que la concepción planteada en la UEA está enmarcada en la cultura tradicional del agua, y el valor de tres, por el contrario, que está enmarcada en la NCA. Lógicamente, el valor numérico 2 representa un estado intermedio.

\section{Descripción de la muestra de libros}

La muestra finalmente utilizada está formada por 10 textos españoles y 10 textos colombianos (ver Anexo 2) seleccionados por ser las editoriales empleadas con más frecuencia en sus contextos respectivos. Concretamente, identificamos los más usados, por un lado, en la ciudad de Melilla (España) y por otro, en el área urbana de Bogotá (Colombia).

El nivel educativo de los manuales es $3^{\circ}$ de Educación Secundaria Obligatoria, en el caso de los españoles, y $9^{\circ}$ de Educación Básica Secundaria, en el de los colombianos, ambos correspondientes a estudiantes de 14-15 años de edad.

En cuanto a las asignaturas, en el caso de los libros españoles, cinco de ellos fueron de Biología y Geología y otros cinco fueron de Geografía e Historia. En los libros colombianos, cinco fueron de Ciencias Naturales y otros cinco de Ciencias Sociales. Estas decisiones fueron tomadas tras un análisis previo de los currículos de ambos países, que mostró que eran las asignaturas de secundaria que absorbían mayor carga curricular sobre la cultura del agua.

\section{Procedimiento}

En primer lugar, procedimos a seleccionar las UEA sobre la cultura del agua, consideradas estas como las unidades de contenido que están relacionadas con cualquiera de los apartados contemplados en la taxonomía de análisis. Para incrementar la objetividad de este proceso de selección, se realizó una fase de entrenamiento en sesiones de videoconferencia, con los cuatro autores de este trabajo y una colaboradora externa, a partir de un manual colombiano elegido al azar. En esta fase, se encontraron algunas dificultades que tuvieron que ser resueltas por la vía del consenso. Así, por ejemplo, se acordó que no fuera la presencia de la palabra "agua" la principal evidencia de la existencia de una UEA; un texto podía estar refiriéndose al agua (como recurso natural, como fuente o sumidero de desechos y de contaminación...) sin que explícitamente se citara dicho término. Por el contrario, su presencia en las unidades de contenido desde una perspectiva informativa (por ejemplo, el agua está formada por hidrógeno y oxígeno, el agua tiene unas propiedades diferentes...) en muchas ocasiones no implicaba una transmisión cultural del agua. 
Una vez seleccionadas las UEA, procedimos a adjudicar un indicador taxonómico a cada una de ellas. Este proceso fue realizado por los cinco investigadores participantes, en primer lugar, de forma individual. Posteriormente, se realizaron varias reuniones por videoconferencia para resolver las discrepancias. Considerando como tales las UEA para las que no existía un acuerdo mínimo de cuatro evaluaciones sobre cinco, el porcentaje de discrepancias alcanzó el valor global del 38\%. Estas discrepancias se resolvieron tras el debate entre las diversas posturas y la defensa argumentativa de cada una de ellas hasta llegar a alcanzar la mayoría exigida del consenso del $80 \%$. Conviene aclarar que, tras el consenso, todas las UEA quedaron adscritas a un único subcontexto.

\section{Resultados}

El número de UEA identificadas en los libros de texto españoles y colombianos se muestra en la Tabla 2. En el primer caso, han sido 157 UEA y, en el segundo, 104, las que han sido seleccionadas y analizadas. Llama la atención, ante todo, la alta dispersión, no solo entre países, sino entre asignaturas de un mismo país. Concretamente, las horquillas de UEA son [19-39] para los manuales españoles de Geografía e Historia; [2-18] para los de Biología y Geología; [3-29] para los manuales colombianos de Ciencias Sociales; y [1-7] para los de Ciencias Naturales. Estos resultados tan variados en el número de UEA incluso entre manuales de un mismo país, indican que, aun cuando hay un currículo común, los autores hacen desarrollos particulares y realizan transmisiones de contenidos muy distintas. Particularmente, llama la atención la escasa transmisión cultural que se realiza desde la enseñanza-aprendizaje de la Biología y Geología española, así como desde las Ciencias Naturales colombianas. Nos preguntamos si es que las Ciencias Experimentales no son portadoras de valores culturales.

TABLA 2

Número de UEA identificadas en los libros de texto españoles y colombianos

\begin{tabular}{|c|c|c|c|}
\hline \multicolumn{2}{|c|}{ Libros españoles } & \multicolumn{2}{|c|}{ Libros colombianos } \\
\hline $\begin{array}{l}\text { Geografía e } \\
\text { Historia. }\end{array}$ & $\begin{array}{l}\text { Biología y } \\
\text { Geología }\end{array}$ & $\begin{array}{l}\text { Ciencias } \\
\text { Sociales }\end{array}$ & $\begin{array}{l}\text { Ciencias } \\
\text { Naturales }\end{array}$ \\
\hline Libro 1: 27 & Libro 6: 2 & Libro 1: 3 & Libro $6: 7$ \\
\hline Libro 2: 39 & Libro 7: 5 & Libro 2: 26 & Libro 7: 5 \\
\hline Libro 3: 20 & Libro 8: 5 & Libro 3: 10 & Libro 8: 5 \\
\hline Libro 4: $\mathbf{1 9}$ & Libro 9: 3 & Libro 4: 13 & Libro 9: 1 \\
\hline Libro 5: 19 & Libro $10: 18$ & Libro 5: 29 & Libro 10: 5 \\
\hline Total: 124 & Total: 33 & Total: 81 & Total: 23 \\
\hline
\end{tabular}

\section{Análisis de Libros de Texto españoles}

Centrándonos primero en los libros españoles, el número de subcontextos y niveles taxonómicos encontrados, se muestra en la Tabla 3. La última columna aloja la media ponderada, un valor numérico global del libro, que indica su tendencia más o menos cercana a la NCA. Una media ponderada de uno es indicativa de un libro transmisor de una vieja cultura del agua y una media de tres sería todo lo contrario, un libro alineado con una NCA. 
TABLA 3

Subcontextos y niveles taxonómicos sobre la NCA tratados en los libros de texto españoles

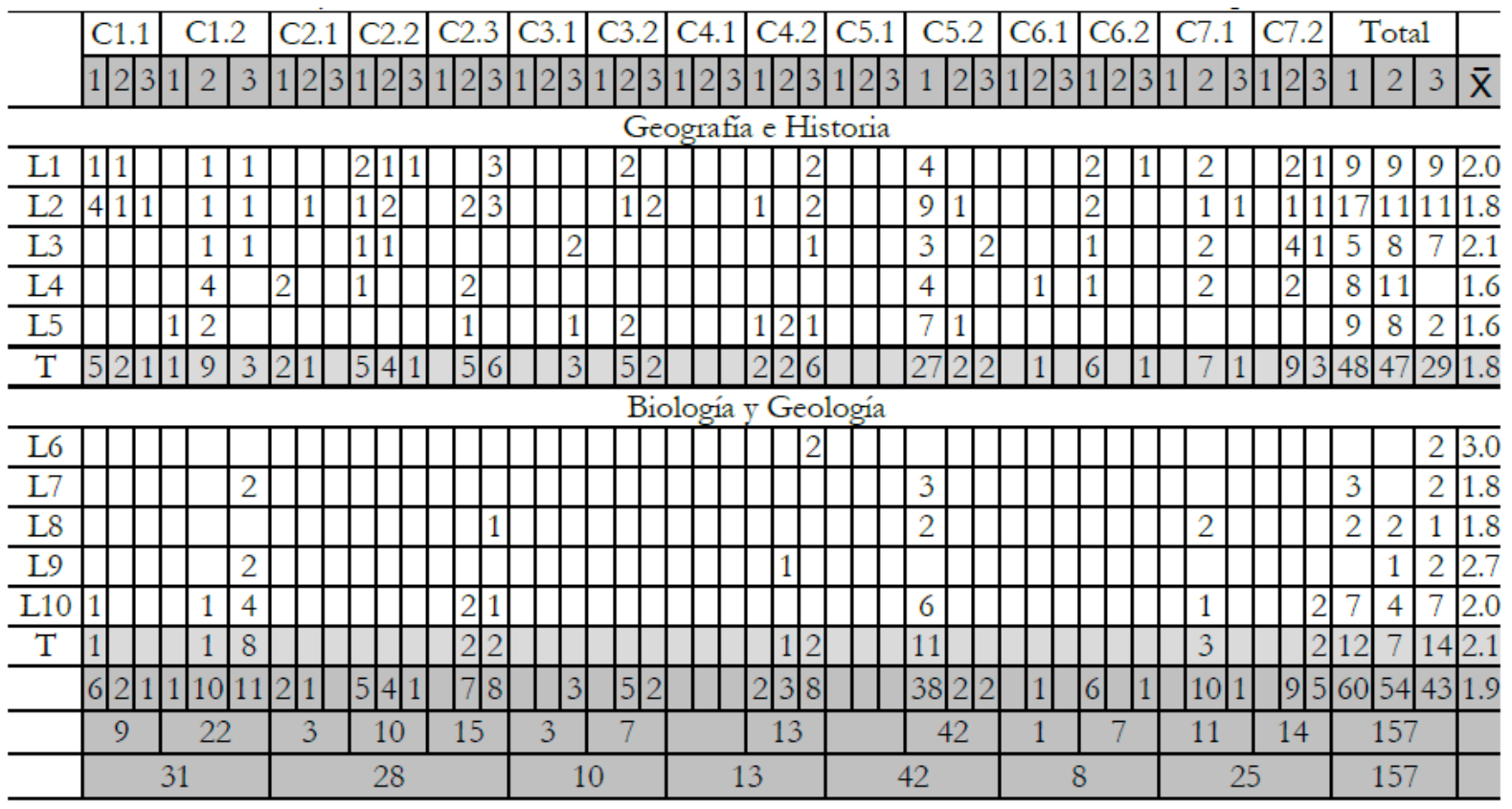

TABLA 4

Síntesis de contextos y niveles taxonómicos sobre la NCA tratados en los libros de texto españoles.

\begin{tabular}{|c|c|c|c|c|c|c|c|c|c|c|c|c|c|c|c|c|c|c|c|c|c|}
\hline & \multicolumn{3}{|c|}{ C1 } & \multicolumn{3}{|c|}{$\mathrm{C} 2$} & \multicolumn{2}{|l|}{$\mathrm{C3}$} & \multicolumn{2}{|c|}{ C4 } & \multicolumn{3}{|l|}{ C5 } & \multicolumn{2}{|l|}{ C6 } & \multicolumn{3}{|c|}{ C7 } & \multicolumn{3}{|c|}{ TOTAL } \\
\hline & 1 & 2 & 3 & 1 & 2 & 3 & \begin{tabular}{l|l}
1 & 2 \\
\end{tabular} & 3 & \begin{tabular}{|l|l}
1 & 2 \\
\end{tabular} & 3 & 1 & 2 & 3 & 1 & 23 & \begin{tabular}{l|l}
3 & 1 \\
\end{tabular} & \begin{tabular}{l|l}
1 & 2 \\
\end{tabular} & 3 & 1 & 2 & 3 \\
\hline $\begin{array}{l}\text { Geografía e } \\
\text { Historia }\end{array}$ & 6 & 11 & 4 & 7 & 10 & 7 & 5 & 5 & 2 & 6 & 27 & 2 & 2 & 6 & \begin{tabular}{l|l}
1 & 1
\end{tabular} & & 16 & 4 & 48 & 47 & 29 \\
\hline $\begin{array}{l}\text { Biología y } \\
\text { Geologia }\end{array}$ & 1 & 1 & 8 & & 2 & 2 & & & 1 & 2 & 11 & & & & & & 3 & 2 & 12 & 7 & 14 \\
\hline Total & 7 & 12 & 12 & 7 & 12 & 9 & \begin{tabular}{l|l}
0 & 5 \\
\end{tabular} & 5 & \begin{tabular}{l|l}
2 & 3 \\
\end{tabular} & 8 & 38 & 2 & 2 & 6 & \begin{tabular}{l|l}
1 & 1 \\
\end{tabular} & 10 & \begin{tabular}{l|l}
0 & 19 \\
\end{tabular} & 6 & 60 & 54 & 43 \\
\hline & 31 & & & 28 & & & 10 & & 13 & & 42 & & & 8 & & & 25 & & 157 & & \\
\hline
\end{tabular}

Entre los manuales españoles, hay uno (L6, de Biología y Geología) que tiene un tres de media ponderada, pero justamente este valor es muy poco significativo porque en dicho manual solo se encontraron dos UEA. Los resultados para los manuales de Geografía e Historia, que son bastante más significativos, arrojan una media ponderada de 1.8 lo que significa que, globalmente, son transmisores de una cultura algo más cercana de la vieja que de la NCA. Además, la dispersión entre los manuales de esta asignatura no es muy alta, pues las medias oscilan en la horquilla [1.6-2.1].

Un análisis de mayor interés es el que se relaciona con los contextos trabajados. El detalle de este análisis para los libros españoles se muestra en la Tabla 4. Por orden de prevalencia, estos son: C5, C1, C2, C7, C4, C3 y C6. Algunos resultados de interés de esta tabla son:

. El contexto más abundante es el número 5, en el subcontexto 5.2 (“Quien contamina, paga”), relacionado con las 42 UEA que narran la existencia de factores contaminantes en el agua, sin hacer referencia a las penalizaciones por descontaminación, que es lo que cabría esperar bajo una perspectiva de la NCA.

- Le sigue el contexto número 1, con 31 UEA, que son las veces que encontramos UEA que aluden al desequilibrio hídrico de la naturaleza. En este caso, afortunadamente, la tendencia más frecuente es la de la NCA, al considerar que el agua puede llegar a ser un recurso no renovable si no se tiene cuidado con su uso y calidad. 
- A continuación, el C2 agrupa 28 UEA, destinados principalmente a los subcontextos 2.2. y 2.3, esto es, a la valoración del agua y a actividades de reflexión crítica sobre la importancia del agua para la vida, respectivamente. En el subcontexto 2.2. es más frecuente una valoración del agua desde la perspectiva exclusivamente económica, lo que se alinea con una vieja cultura del agua. Un ejemplo se muestra en la Figura 1, extraído del Libro 1 (UEA 11). En el subcontexto 2.3, en cambio, las actividades de reflexión tratan de destacar la importancia del agua para las personas desde múltiples puntos de vista, alineándose con la NCA. Un ejemplo se muestra en la Figura 1, extraído también del Libro 1 (UEA 15).

- Los contextos C7 (con 25 UEA), C4 (con 13 UEA) y C3 (con 10 UEA) son trabajados en los libros de texto españoles con tendencia moderada hacia la NCA. Esto significa que se fomenta el ahorro del agua y el consumo responsable (contexto 7), se destacan los impactos medioambientales de las obras hidráulicas (contexto 4) y se responsabiliza a la ciudadanía de la gestión del agua, según movimientos sociales que promueven la NCA (contexto 3).

- Por último, el contexto número 6 (con 8 UEA) tiene por el contrario una tendencia generalizada hacia la cultura del agua tradicional, pues se hace referencia a la existencia de poblaciones que carecen de agua potable y/o servicios de saneamiento básicos, pero de forma descontextualizada y alejada de la realidad del estudiante.

Conviene destacar que los contextos C4.1 y C5.1 no aparecen en los libros de texto. En otras palabras, en ellos no se plantea la disyuntiva entre la ampliación de la oferta o la demanda del agua ante el exceso de su consumo (C4.1), ni se referencian los costes diferenciados del agua según sus usos (C5.1).
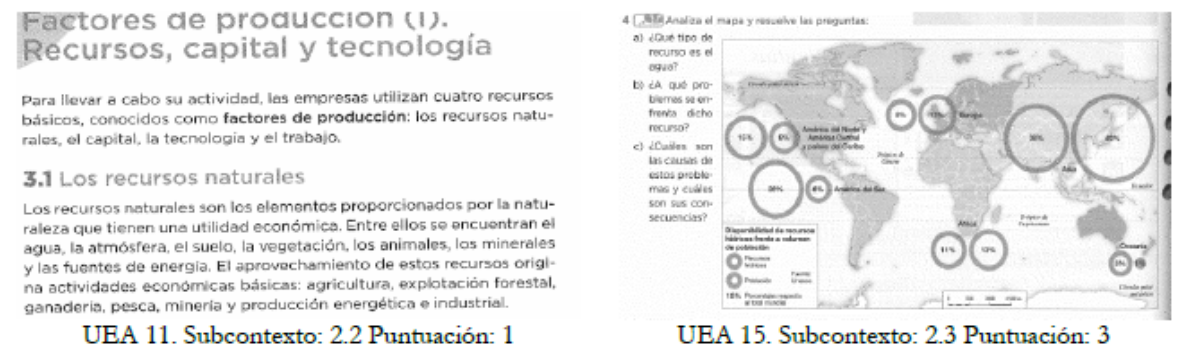

FIGURA 1

Ejemplos de UEA de los subcontextos 2.2 y 2.3. con tendencias culturales opuestas en un mismo manual (Libro 1 de Geografía e Historia español)

\section{Análisis de Libros de Texto colombianos}

Centrándonos ahora en los libros colombianos, el número de subcontextos y niveles taxonómicos encontrados, se muestra en la Tabla 5. La última columna aloja la media ponderada, que en este caso es de 1.9 lo que significa que, de nuevo, estos manuales son también transmisores de una cultura ligeramente más cercana de la vieja o tradicional que de la nueva. No obstante, en este caso la dispersión es algo más alta. Si se incluyen los libros de Ciencias Naturales, las medias oscilan en la amplia horquilla [1.2-2.7]. Cabe preguntarse cuál es el motivo de tan alta dispersión. ¿Acaso se refleja la imagen cultural de los propios autores? 
TABLA 5

Subcontextos y niveles taxonómicos sobre la NCA tratados en los libros de texto colombianos

\begin{tabular}{|c|c|c|c|c|c|c|c|c|c|c|c|c|c|c|c|c|c|c|c|c|}
\hline & .1 & $\mathrm{C} 1.2$ & & $\mathrm{C} 2.1$ & $\mathrm{C} 2.2$ & $\mathrm{C} 2.3$ & $\mathrm{C} 3.1$ & $\mathrm{C} 3.2$ & C4.1 & C4.2 & C5.1 & \begin{tabular}{c|}
$\mathrm{C} 5.2$ \\
\end{tabular} & \multirow{2}{*}{\multicolumn{2}{|c|}{\begin{tabular}{|l|l|} 
C6.1 & C6.2 \\
\end{tabular}}} & \multirow{2}{*}{\multicolumn{2}{|c|}{$\mathrm{C} 7.1$}} & \multicolumn{4}{|c|}{ Total } \\
\hline & 23 & 2 & 3 & $12 \sqrt{3}$ & \begin{tabular}{l|ll}
1 & 2 & 3 \\
\end{tabular} & \begin{tabular}{|l|l|}
1 & 2 \\
\end{tabular} & $12 \sqrt{3}$ & 1123 & \begin{tabular}{l|l|l|}
1 & 2 & 3 \\
\end{tabular} & 123 & \begin{tabular}{l|l|l|}
1 & 2 & 3 \\
\end{tabular} & \begin{tabular}{l|l|l|}
1 & 2 \\
\end{tabular} & & & & & 1 & 2 & 3 & $\overline{\mathrm{X}}$ \\
\hline & & & & & & & & & iencia & $\overline{\mathrm{ci}}$ & iales & & & & & 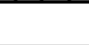 & & & & \\
\hline 1 & & & 1 & & & & & & & & & 1 & & & & & 1 & 11 & \begin{tabular}{|l|}
1 \\
\end{tabular} & 2.0 \\
\hline L2 & & & & & 1 & 5 & 1 & 131 & & & & 4 & 1 & & 1 & 3 & 7 & 14 & 5 & 1.9 \\
\hline $\mathrm{L3}$ & & & & & & 1 & & & & & & 2 & \begin{tabular}{l|l|}
1 & 1 \\
\end{tabular} & & & 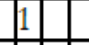 & 2 & 5 & 3 & 2.1 \\
\hline L4 & & & 1 & 1 & $\begin{array}{ll}3 & 1 \\
\end{array}$ & & 1 & 11 & & & & 1 & & & & & 4 & \begin{tabular}{|l|}
4 \\
\end{tabular} & 5 & 2.1 \\
\hline L & & 1 & & & 321 & 52 & & 112 & & & & 4 & 31 & & 1 & & 12 & 13 & 4 & 1. \\
\hline $\mathrm{T}$ & & \begin{tabular}{|l|l}
1 & \\
\end{tabular} & 2 & & 742 & 1112 & 23 & 262 & & 1 & & \begin{tabular}{|l|l|}
1 & 2 \\
\end{tabular} & 242 & & 1 & 32 & 26 & 37 & & 1. \\
\hline & & & & & & & & & & יודר & & & & & & & 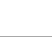 & & & \\
\hline L6 & 1 & & & & {$[1$} & 11 & & & & & & 1 & & & & ]$_{2}$ & & 2 & 5 & 2.7 \\
\hline $\mathrm{L} /$ & & & & & & & & & & 22 & & 1 & & & & & 3 & 2 & $\square$ & 1 \\
\hline L8 & 1 & & & & & 2 & & & & & & 1 & & & & & 1 & 3 & 1 & 2 \\
\hline L9 & & & & & & 1 & & & & & & & & & & & & 1 & 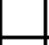 & 2. \\
\hline L10 & 1 & & & & & & & & & & & 2 & & & & & 4 & 1 & & 1.2 \\
\hline $\mathrm{T}$ & 21 & & & & & 4 & & & & 22 & & 4 & & & & & 88 & 9 & \begin{tabular}{|l|}
6 \\
\end{tabular} & 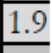 \\
\hline & 121 & 11 & 2 & 211 & $7 \sqrt[4]{3}$ & 15 & 23 & 2262 & & \begin{tabular}{|l|l|l|}
3 & 3 & 1 \\
\end{tabular} & & 143 & 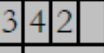 & 31 & 12 & 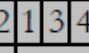 & 34 & 46 & 24 & 1. \\
\hline & 4 & 3 & & 3 & 14 & 17 & 5 & 10 & & 7 & & 20 & 6 & 4 & 3 & 8 & & 104 & & \\
\hline & & 7 & & & 34 & & & 15 & & 7 & & 20 & & 10 & 1 & 11 & & 104 & & \\
\hline
\end{tabular}

TABLA 6

Síntesis de contextos y niveles taxonómicos sobre la NCA tratados en los libros de texto colombianos

\begin{tabular}{|c|c|c|c|c|c|c|c|c|c|c|c|c|c|c|c|c|c|c|c|c|c|c|c|}
\hline & \multicolumn{3}{|c|}{$\mathrm{C} 1$} & \multicolumn{3}{|c|}{$\mathrm{C} 2$} & \multicolumn{3}{|c|}{$\mathrm{C} 3$} & \multicolumn{3}{|c|}{$\mathrm{C} 4$} & \multicolumn{3}{|c|}{$\mathrm{C} 5$} & \multicolumn{3}{|c|}{$\mathrm{C} 6$} & \multicolumn{2}{|c|}{ C7 } & \multicolumn{3}{|c|}{ TOTAL } \\
\hline & 1 & 2 & 3 & 1 & 2 & 3 & \begin{tabular}{|l|} 
\\
\end{tabular} & 2 & \begin{tabular}{|l|}
3 \\
\end{tabular} & 1 & \begin{tabular}{|l|}
2 \\
\end{tabular} & 3 & 1 & 2 & 3 & 1 & \begin{tabular}{|l|l|}
2 & \\
\end{tabular} & 3 & \begin{tabular}{|l|l}
1 & \\
\end{tabular} & \begin{tabular}{l|l}
2 & 3 \\
\end{tabular} & 1 & 2 & 3 \\
\hline $\begin{array}{l}\text { Ciencias } \\
\text { Sociales }\end{array}$ & 1 & 1 & 2 & 7 & 16 & 4 & 2 & 8 & 5 & 1 & 1 & 1 & 10 & 2 & 2 & 4 & 5 & 1 & 1 & \begin{tabular}{l|l}
4 & 3
\end{tabular} & 26 & 37 & 18 \\
\hline $\begin{array}{c}\text { Ciencias } \\
\text { Naturales } \\
\end{array}$ & & 2 & 1 & 2 & 4 & 1 & & & & 2 & 2 & & 4 & 1 & 1 & & & & & 3 & 8 & 9 & 6 \\
\hline Total & 1 & \begin{tabular}{|l|}
3 \\
\end{tabular} & 3 & 9 & 20 & 5 & 2 & 8 & 5 & 3 & \begin{tabular}{|l|}
3 \\
\end{tabular} & 1 & 14 & 3 & 3 & 4 & 5 & 1 & \begin{tabular}{|l|}
1 \\
\end{tabular} & \begin{tabular}{l|l}
4 & 6
\end{tabular} & 34 & 46 & 24 \\
\hline
\end{tabular}

El detalle de los contextos trabajados para los libros colombianos se muestra en la Tabla 6. Por orden de prevalencia, estos son: C2, C5, C3, C7, C6 y quedan equiparados al final, C1 y C4. Algunos resultados de interés de esta tabla son:

- El contexto más abundante es el número 2, con 34 UEA, distribuidas en el subcontexto 2.1 (3 UEA), en el subcontexto 2.2 (14 UEA) y en el subcontexto 2.3 (17 UEA). El más frecuente es este último ("Actividades de reflexión crítica sobre la importancia del agua para la vida"), con 17 UEA, que reflejan las veces que hemos encontrado actividades de reflexión crítica sobre la importancia del agua para la vida.

- Le sigue el contexto número 5, con 20 UEA, concentradas en el subcontexto 5.2, y en su mayor parte (14 de 20 UEA) citan la existencia de factores contaminantes y el impacto en el medio ambiente, sin referencia a los costes económicos de la descontaminación, como cabría esperar desde la NCA. Un ejemplo se muestra en la mitad izquierda de la Figura 2 (UEA 11).

- A continuación, el C3 agrupa 15 UEA, distribuidos entre el subcontexto 3.1 (5 UEA) y el 3.2 (10 UEA). Si bien, en este último contexto, hay el mismo número de UEA tradicionales y novedosas, en el primer subcontexto, se produce una ligera tendencia hacia la NCA, al contemplar la participación ciudadana en la gestión del agua. Un ejemplo se muestra en la mitad derecha de la Figura 2 (UEA 15). 
- El C7 agrupa 11 UEA, también distribuidas entre los dos subcontextos 7.1 y 7.2, aunque en este caso en ambos hay un número mayor de UEA de la NCA que de la tradicional. Un ejemplo de UEA de la NCA en cada uno de estos subcontextos se muestra en la Figura 3.

- El C6 cuenta con 10 UEA, 6 de ellas para el subcontexto 6.1 y 4 para el subcontexto 6.2. Cabe destacar la presencia de los primeros en los libros colombianos, a diferencia de lo que vimos que ocurría con los españoles donde el subcontexto 6.1 no aparecía. En este caso, lo hace para hablar sobre todo de los derechos humanos respecto al agua, aunque no se suele asociar a deberes. En cuanto al subcontexto 6.2, relacionado con la solidaridad frente a carencias de agua, en la mayor parte de las ocasiones se hace alusión a este problema de forma descontextualizada y alejada de la realidad del estudiante.

- $\mathrm{C} 1$ y C4 comparten un número de 7 UEA cada uno. Las 7 UEA del C1 se reparten entre los subcontextos 1.1 y 1.2 , con cierta tendencia en este último hacia la NCA, al afirmar que el agua puede llegar a ser un recurso no renovable si no se controla su consumo y su calidad. Las 7 UEA del C4 se concentran en el subcontexto 4.2, donde hay una ligera mayoría de UEA alineadas con la cultura tradicional del agua frente a la NCA. Esto se debe a que se contemplan los beneficios de las grandes obras hidráulicas y no sus impactos ambientales.

Conviene destacar que los contextos C4.1 y C5.1 no aparecen en los libros de texto colombianos, del mismo modo que vimos que ocurría con los españoles. Esto es, vuelve a quedar en el ostracismo la disyuntiva entre la ampliación de la oferta o la demanda del agua ante el exceso de su consumo (C4.1), y las referencias a los costes diferenciados del agua según sus usos (C5.1).

\section{El extractivismo en Colombia}

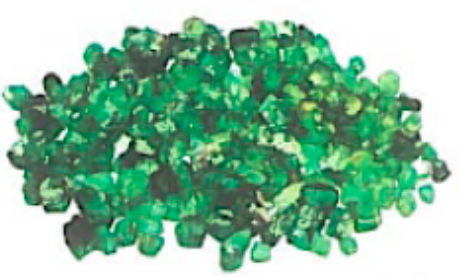

La mineria de esmeraldas se desarolla principalmente en Boyací.

El extractivismo es un modelo de desarrollo económico basado en una alta dependencia de la extracción intensiva de los recursos naturales. Colombia, desde el 2008, ha impulsado el sector mineroenergético principalmente por medio de la inversión extranjera con la explotación del carbón, el níquel, los metales precio505, las esmeraldas y el hierro. Sin embargo, las actividades mineras han generado impactos ambientales fuertes debido al uso de productos tóxicos que contaminan el suelo y las fuentes hidricas. Además, para los proyectos mineros a gran escala, se cambian los cursos de los ríos, se deforesta el área a explotar y se utilizan enor. mes cantidades de explosivos.

UEA 11 Subcontexto 5.2. Puntuación: 1

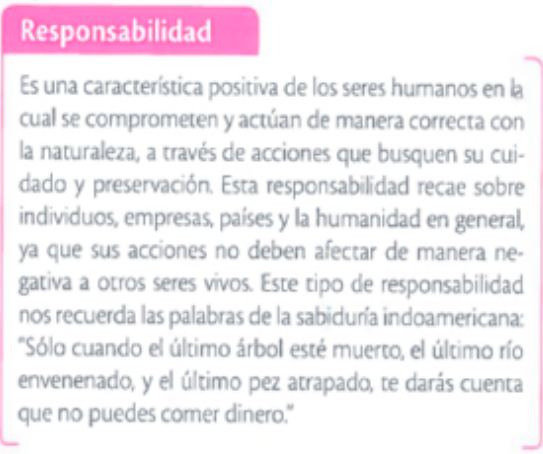

UEA 15. Subcontexto 3.1. Puntuación: 3

FIGURA 2

Ejemplos de UEA de los subcontextos 5.2 y 3.1 con tendencias culturales opuestas en un mismo manual (Libro 5 de Ciencias Sociales colombiano) 


\section{Conclusiones y Discusión de Resultados}

Uno de los resultados más llamativos de este estudio es la alta dispersión en la imagen cultural que sobre el agua ofrecen los libros de texto, tanto españoles como colombianos. Esta dispersión se manifiesta no solo entre países distintos sino incluso dentro de cada país. Tanto es así, que no podemos afirmar de forma contundente cuál es la tendencia más marcada que transmiten los libros de texto. Cada libro transmite una imagen particular acerca de la cultura del agua y contempla contextos y subcontextos distintos.

A pesar de lo anterior, es posible destacar globalmente la existencia de UEA con propensiones equilibradas hacia ambas culturas, la tradicional y la nueva, con cierta desviación proporcional hacia la tradicional. De hecho, las 157 UEA identificadas en los libros españoles se distribuyen bien entre los sucesivos indicadores que conforman la progresión desde la cultura tradicional a la nueva cultura del agua, según cifras (60, 54 y 43, respectivamente) que indican solo cierta tímida tendencia hacia la cultura más tradicional. En el caso de los libros colombianos, la distribución de las 104 UEA (34, 46 y 24) es asimismo bastante equitativa entre los sucesivos indicadores, pero también se manifiesta la tímida tendencia hacia la cultura más tradicional.

Tampoco hemos encontrado grandes diferencias entre los libros de texto españoles y colombianos. Se esperaba que estas hubieran sido mayores entre dos países con niveles de estrés hídrico tan diferentes, pero no ha sido así. En los manuales de ambos países, la variedad es la tendencia generalizada y la falta de una apuesta contundente, también.

¿Cuál puede ser el motivo de que no haya una postura definida? Pensamos que esto podría ser una consecuencia de la falta de un debate más amplio entre la sociedad y los gobiernos, e incluso de la falta de una postura ideológica concreta en los currículos oficiales de los respectivos países, lo que podría conducir a los autores de los libros de texto a promover posturas también amorfas y eclécticas, que no entren en conflicto con las opiniones más extendidas (Pérez y Meneses 2020).

La cultura del agua es un objeto de estudio interdisciplinar, por lo que en este trabajo se han revisado textos de Ciencias Naturales y de Ciencias Sociales tanto españoles como colombianos. En ambos países, el número de UEA identificadas para Ciencias Naturales ha sido muy inferior al de Ciencias Sociales. Nos preguntamos si los libros de Ciencias Naturales son portadores de una pobre visión acerca de los valores culturales del agua o si se están mostrando a los estudiantes de una forma menos contextualizada y aséptica culturalmente frente a los libros de Ciencias Sociales.

Otra reflexión que surge de los resultados de este trabajo es la relacionada con los contextos que sobre la cultura del agua se trabajan en los libros de texto. Aun cuando aquí sigue prevaleciendo la máxima de la diversidad y la ausencia de patrones comunes, incluso dentro de cada país, hay ciertas tendencias que merece la pena destacar.

Una de ellas es la alta frecuencia de los contextos C2 y C5, tanto en los libros españoles como en los colombianos. Para el primero de ellos, no se puede afirmar que la imagen del agua que se transmite en los libros de texto sea exclusivamente económica, si bien tampoco se le llega a otorgar el valor patrimonial y emocional que se le adjudica desde la NCA (Declaración Europea por una Nueva Cultura del Agua 2005, p.12). En el caso del C5, es muy abundante el número de veces que se menciona la contaminación de las aguas sin alusión al principio contaminador-pagador, como cabría esperar desde la NCA (Declaración Europea por una Nueva Cultura del Agua 2005, p.38).

Asimismo, también hay ciertas tendencias comunes entre los libros de texto españoles y colombianos en cuanto a los resultados globales asociados a los criterios C1, C3 y C7. Para ellos, se ha obtenido una cierta frecuencia mayoritaria de los indicadores asociados a la NCA. En consecuencia, valoramos que los libros destaquen ideas que encierran concepciones tan importantes como las siguientes: la naturaleza no está mal hecha, somos los seres humanos los que hemos de acoplarnos a ella (C1); la ciudadanía es corresponsable de la gestión del agua y debe implicarse activamente en ella (C3); y son muchas las actividades y hábitos que se 
pueden adoptar para reducir el consumo de agua, a todos los niveles, incluido el más personal relacionado con nuestros hábitos consumistas y alimentarios (C7).

Otra cuestión que surge de nuestros resultados, es que los libros de texto no contemplan determinados contenidos, concretamente, los asociados a los subcontextos C4.1 y C5.1. El primero está relacionado con la disyuntiva entre incrementar la oferta de agua o reducir la demanda, que es una controversia de enorme actualidad y sumamente necesaria ante la problemática del consumo desorbitado de agua. Por ejemplo, según datos de 2017, el consumo de agua en España sigue siendo uno de los más altos de Europa (Aquastat-FAO, 2017). ¿No debería ser de interés para los estudiantes españoles un planteamiento donde pudieran valorar distintas alternativas y soluciones a esta problemática? En cuanto al contexto 5.1, relacionado con el coste del agua que asume la ciudadanía, como dice Ibarra (2007), se trata de un contenido que podría explicar muy eficazmente la historia de una gestión desafortunada y enseñar que unas nuevas tarifas pueden llevar a proteger y mantener los ecosistemas acuáticos.

En definitiva, no todo está mal en los libros de texto. Al menos, en lo que se refiere a la cultura hídrica, hay ciertos contextos para los que las tendencias más frecuentes se alinean en la NCA, tratando de vencer a la cultura tradicional. Pero otros que no aparecen y otros para los que falta una mayor decisión y una apuesta más firme por una cultura hídrica a favor de un futuro que es el único posible. Así, por ejemplo, debería quedar más claro en los libros de texto, que:

- El consumo de agua se ha disparado y el agua ha dejado de ser un recurso renovable. Hemos de priorizar la preservación del agua que tenemos.

- El agua no es un bien económico, sino que tiene un valor patrimonial importante. La protección de su calidad debe primarse frente a la explotación.

- La responsabilidad del agua es de toda la ciudadanía, del estilo de vida en los países más desarrollados y del crecimiento demográfico en los menos desarrollados.

- Se debe reducir el consumo de agua y no tratar de satisfacer cualquier demanda.

- Las tarifas de agua deben analizarse cuidadosamente de modo que se garantice el derecho al agua-vida, pero se penalice seriamente un consumo excesivo del agua.

- Quien contamina debe dejar de hacerlo (se arriesga a una sanción importante)

- Nuestro estilo de vida (hábitos consumistas y alimenticios) está íntimamente relacionado con el consumo de agua.

\section{Materiales SUPLEMENTARios}

Anexo $1(\mathrm{pdf})$

Anexo 2 (pdf)

\section{ReFERENCIAS}

Antoraz M.A., Martínez F.J. (2003) El agua y el sistema educativo español, pp. 385-424 en L. Del Moral I. y Arrojo P. (coord.), La directiva marco del agua: realidades y futuros. Fundación Nueva Cultura del Agua.

Aquastat-FAO (2017) Global Information System on Water and Agriculture. Food and Agriculture Organization of the United Nations (FAO). http://www.fao.org/aquastat/statistics/query/index.html 
Arrojo P. (2009) El reto ético de la crisis global del agua. Relaciones Internacionales 12, 33-53. https://revistas.uam.e s/relacionesinternacionales/article/view/4979

Benarroch, A., Rodríguez-Serrano, M., Ramírez-Segado, A. (2021). The new water culture versus the traditional. Design and validation of a questionnaire to discriminate between both. Sustainability, 13 (4), 2174. https://d oi.org/10.3390/su13042174

Cañal P., Travé G., Pozuelos F.J., Criado A.M., García A. (2016) La enseñanza sobre el medio natural y social. Díada.

Comunidades Europeas. (2000). Directiva 2000/60/CE del Parlamento Europeo y del Consejo, de 23 de octubre de 2000, por la que se establece un marco comunitario de actuación en el ámbito de la política de aguas. DOUE núm. 327, de 22 de diciembre de 2000, pp. 1-73.

Cuello, A., García Pérez, F. (2019) ¿Ayudan los libros de texto a comprender la realidad fluvial de la ciudad? Revista de Humanidades, 37, 209-234. https://doi.org/10.5944/rdh.37.2019.22895

Fernández M.P., Caballero P.A. (2017) El libro de texto como objeto de estudio y recurso didáctico para el aprendizaje: fortalezas y debilidades. Revista Electrónica Interuniversitaria de Formación del Profesorado 20 (1), 201-217. ht tps://doi.org/10.6018/reifop/20.1.229641.

Fernández-Ferrer G. (2009) El agua subterránea: estudio de esquemas de conocimiento en universitarios y estrategias didácticas para su aprendizaje significativo en estudiantes de secundaria (tesis doctoral). Universidad de Granada, España.

Ibarra J. (2007) Nuevos contenidos educativos sobre el agua y los ríos desde una perspectiva CTS. Revista Electrónica de Enseñanza de las Ciencias 6, 714-728. http://reec.uvigo.es/volumenes/volumen6/ART13_Vol6_N3.pdf

López-Valentín D.M, Guerra-Ramos M.T. (2013) Análisis de las actividades de aprendizaje incluidas en libros de texto de ciencias naturales para educación primaria utilizados en México. Enseñanza de las Ciencias 31 (2), 173-179. https://core.ac.uk/download/pdf/18416354.pdf

Martínez N., Alarcón M.D. (2016) La utilización del libro de texto de Historia de España dentro y fuera del aula: alumnos, manuales, huella, interpretación y contexto. Universidad de Murcia.

Martínez J., Rodríguez J. (2010) El currículum y el libro de texto escolar. Una dialéctica siempre abierta, pp. 246-269 en J. Gimeno (comp.), Saberes e incertidumbres sobre el curriculum. Morata.

Nnebuihe N. (2019) The Efficacy of Dimensional Shingle on National Integration, Development and Sustainability: Education, Culture and Innovation. International Journal of Research Development 11 (1), 1-6. https://www.gl obalacademicgroup.com/journals/approaches/V11N1P14-2019_Approaches.pdf

Occelli M., Valeiras N. (2013) Los libros de texto de ciencias como objeto de investigación: una revisión bibliográfica. Enseñanza de las Ciencias 31 (2), 133-152. https://raco.cat/index.php/Ensenanza/article/view/285774/3737 74

Parcerisa A. (1996) Materiales curriculares. Cómo elaborarlos, seleccionarlos y usarlos. Graó.

Perales F.J. (2019) ¿Cómo podemos ayudar a los maestros en formación a analizar los libros de texto de ciencias? UTE. Revista de Ciències de l'Educació Monogräfic, 33-42. https://doi.org/10.17345/ute.2019.2

Pérez R. (2015) La Nueva Cultura del Agua, el camino hacia una gestión sostenible: causas e impactos de la crisis global del agua. Cuadernos de trabajo Hegoa 68, 1-53. https://ojs.ehu.eus/index.php/hegoa/article/view/15672

Pérez S., Meneses J.A. (2020) La competencia científica en las actividades de aprendizaje incluidas en los libros de texto de Ciencias de la Naturaleza. Revista Eureka sobre Enseñanza y Divulgación de las Ciencias 17 (2), 2101. https:/ /doi.org/10.25267/Rev_Eureka_ensen_divulg_cienc.2020.v17.i2.2101

Ramírez-Segado, A., Rodríguez-Serrano, M., Benarroch, A. (2021). El agua en la literatura educativa de las dos últimas décadas. Una revisión sistemática. Revista Eureka sobre Enseñanza y Divulgación de las Ciencias, 18(1), 1107. h ttps://doi.org/10.25267/Rev_Eureka_ensen_divulg_cienc.2021.v18.i1.1107

Robles I.M., Maldonado L.F. (2018) The culture of water care and consumption habits in Querétaro. CONIIN. XIV Congreso Internacional de Ingeniería, Querétaro. https://www.researchgate.net/publication/336927876_The _culture_of_water_care_and_consumption_habits_in_Queretaro 
Santana A.I., Cabrera M.C., Pérez-Torrado F.J. (2016) Ideas preconcebidas sobre el ciclo del agua y las aguas subterráneas en la educación secundaria de Canarias. II Workshop Estudio, aprovechamiento y gestión del agua en terrenos e islas volcánicas.

Shcwab J. (2017) Israel is the unsung hero in water management. https://www.huffingtonpost.com/jennifer-schwab /israel-is-the-unsung-hero_b_9212810.html

Sutoris P. (2021) The climate crisis requires a new culture and politics, not just new tech. https://www.theguardian.com /commentisfree/2021/may/24/climate-change-crisis-culture-politics-technology

UNESCO (2010) Article 13 of the Convention on the Protection and Promotion of the Diversity of Cultural Expressions. Operational guidelines - Integration of culture in sustainable development. Organización de las Naciones Unidas para la Educación, la Ciencia y la Cultura (UNESCO) http://www.unesco.org/culture/culturaldiversity/artic le13_en.pdf

WWDR (2020) Informe de las Naciones Unidas sobre el desarrollo de los recursos hidricos en el mundo 2020, Agua y cambio climático. Organización de las Naciones Unidas para la Educación, la Ciencia y la Cultura (UNESCO). https://www.unwater.org/publications/world-water-development-report-2020/

WRI (2019) 17 Countries, Home to One-Quarter of the World's Population, Face Extremely High Water Stress. World Resources Institute. https://www.wri.org/insights/17-countries-home-one-quarter-worlds-population-face-ex tremely-high-water-stress

\section{INFORMACIÓN ADICIONAL}

Para citar este artículo: Benarroch, A., Castro-Velásquez, F.E., Clavijo-Cuervo, V.J. y Ramírez-Segado, A. (2022). La cultura del agua en los libros de texto. Revista Eureka sobre Enseñanza y Divulgación de las Ciencias 19(1), 1501.doi: 10.25267/Rev_Eureka_ensen_divulg_cienc.2022.v19.i1.1501 